\title{
Communication Through Motion: Legibility of Multi-Robot Systems
}

\author{
Beatrice Capelli, Cristian Secchi, Lorenzo Sabattini
}

\begin{abstract}
The interaction between a user and a multi-robot system in a shared environment is a relatively uncharted topic. But, as these types of systems will increase in the future years, an efficient way of communication is necessary. To this aim, it is interesting to discover if a multi-robot system can communicate its intentions exploiting only some motion-variables, which are characteristics of the motion of the robots. This study is about the legibility of a multi-robot system: in particular, we focus on the influence of these motion-variables on the legibility of more than one group of robots that move in a shared environment with the user. These motion-variables are: trajectory, dispersion and stiffness. They are generally used to define the motion of a group of mobile robots.

Trajectory and dispersion were found relevant for the correctness of the communication between the user and the multi-robot system, while stiffness was found relevant for the rapidity of communication. The analysis of the influence of the motion-variables was carried out with an ANOVA (analysis of variance) based on a series of data coming from an experimental campaign conducted in a virtual reality set-up.
\end{abstract}

\section{INTRODUCTION}

Human-multi-robot interaction is a new frontier in the robotics field and it is increasingly becoming important because of the growth of popularity of multi-robot systems that share the same environment with human users. For example, consider automated guided vehicle inside automatic warehouses and small-size multi-robot systems (ground, aerial, underwater, etc.), which will be widespread also in domestic applications in the near future.

The survey about human-swarm-interaction [1] points out the low development of methods of communication in the case of proximal interaction, which implies a shared environment between the user and the multi-robot system. Meanwhile, there exist a lot of studies about remote interaction [2]-[4], in which the two agents do not share the same environment.

In this paper, we focus on proximal interaction and in the direct communication between users and robots. There exist studies that cover both directions of communication: from the user to the robot and vice-versa. In fact, the user should be able to control the robotic system and, taking a cue from the communication between humans, a simple way is to implement speech, or face and gesture recognition [5], [6].

On the other side, the multi-robot system should be able to report to the user useful information about its status (e.g., battery remaining, eventual errors) or about its current task

\footnotetext{
Authors are with the Department of Sciences and Methods for Engineering (DISMI), University of Modena and Reggio Emilia, Italy \{beatrice.capelli, cristian.secchi, lorenzo.sabattini\}@unimore. it
}

(e.g., state of progress, need of help from the user) or about its intention (e.g, next goal, next task). All these aspects can be incorporated inside legibility, which is the ability of a robot, or of a group of robots, to communicate its intention to the user. Also in this case, multiple methods have been developed to allow communication. These methods can be divided, along the lines of [7], in explicit cues and implicit cues. In this paper, we focus on implicit cues, that involve direct use of the robots to convey information: namely, they communicate through their motion [8]-[10].

In this paper, we want to discover if a set of motionvariables affects the legibility of multi-robot system, in particular composed of more than one group of robots. This work extends our previous work [11] in which we have investigated the legibility of a single group of mobile robots. We believe that the results obtained for a single group can not be directly scaled to the case of more groups. A more tailored study is needed to discover if the same motionvariables affect the legibility and, in that case, if the same combination of variables results as the most legible. This additional research is necessary because a multi-robot system is very complex and its complexity grows with the number of robots and with the number of groups of robots. Specifically, in our previous work, the user should focus only on a group (complexity $O(1)$ ), while during the experiments conducted in this work she/he should focus on $n$ groups (complexity $O(n))$. Hence, with the growth of the complexity of the system, we can not state that the results retrieved from the proximal interaction with one group of robots also apply to the case of multiple groups.

\section{DEFINITION}

Along the lines of our previous work [11], we can define legibility ${ }^{1}$ as the ability of a multi-robot system to convey information to the user exploiting implicit cues, namely without using direct means of communication, such as speech or color patterns. In this paper, we want to extend the definition of legibility of a group of mobile robots to more groups of robots. In order to discover if a certain set of motionvariables affects the legibility of a multi-robot systems, we should introduce a function that represents the probability of understanding the goals of the different groups that share the working area alongside with the user. This function is called inference function and, retrieving its definition from our previous work [11], it is possible to scale it to a more complex system.

\footnotetext{
${ }^{1}$ Legibility is also defined for a manipulator in [10].
} 
If we consider a multi-robot system composed by $N$ mobile robots, divided into $Q$ groups, and we focus on a single group $q \in Q$, we can define the inference function for the single group as:

$$
\mathcal{I}_{G O A L}:\left(\Upsilon_{q}\right) \rightarrow \mathcal{G}
$$

where $\Upsilon_{q}$ represents the state of the group, namely the set of motion-variables that characterize its motion, and $\mathcal{G}$ is the set of possible goals in the environment.

If we want to consider all the groups $Q$ together, we should consider the overall state of the system $\left(\Upsilon_{Q}\right)$ and we can define the inference function for the entire multi-robot system as:

$$
\mathbb{I}_{G O A L}:\left(\Upsilon_{Q}\right) \rightarrow \mathfrak{G}
$$

where $\mathfrak{G}$ is a family of functions that map all the groups, and hence all the robots, in their respective goals:

$$
\mathfrak{G}: N \rightarrow \mathcal{G}
$$

The state of the system $\Upsilon_{Q}$ is represented by the motionvariables of which we want to discover the inference over legibility:

- $\mathcal{T}$ : trajectory of the center of the group;

- $\mathcal{D}$ : dispersion of the group;

- $\mathcal{S}$ : stiffness of the group.

Each of these variables can be defined for each group and hence they define the overall behavior of the system. These variables have been chosen because they are useful to define the motion of each group of robots. Trajectory is a fundamental characteristic of the motion because it defines the path that the robot follows from the starting point to the ending point. Meanwhile, dispersion and stiffness allow to define the cohesiveness, synchronization and the general aspect of a formation of robots. Trajectory has already been studied in [10], while synchronization has been investigated in [8].

The inference function represents the most probable function $\mathbb{G}$ that maps all the groups of robots in their respective goals:

$$
\mathbb{I}_{G O A L}\left(\Upsilon_{Q}\right)=\arg \max _{\mathbb{G} \in \mathfrak{G}} P\left(\mathbb{G} \mid \Upsilon_{Q}\right)
$$

It is worth noting that, differently from the one group case, the inference function $\mathbb{I}_{G O A L}$ maps the state of the system $\left(\Upsilon_{Q}\right)$ to a function $(\mathbb{G})$ and not to a single goal. This definition incorporates the complexity of having more than one group in the same environment.

\section{METHODS}

The influence of the motion-variables over the legibility of a multi-robot system composed by multiple groups has been investigated through experiments carried out in a virtual reality set-up.

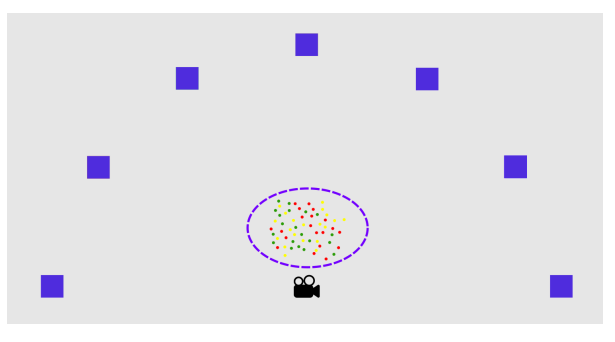

(a) Top-down view of the position of the goals (colored cubes) and of the robots (circled in blue) with respect to the user (camera icon).

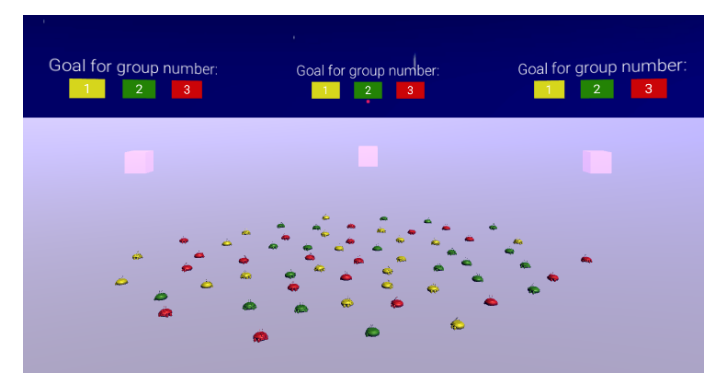

(c) Interface for the choice of the answers.

Fig. 1: Virtual reality environment.

\section{A. Aim of the experiment}

The different combinations of motion-variables have been investigated in different trials. In every trial, all the groups moved with the same configuration towards a random goal. The same goal could be shared among different groups. The combination has been evaluated by the users, who were asked to understand, in the correct and fastest way, where each group of robots was moving (i.e., what was the goal).

\section{B. Experimental scenario}

To test the influence of the motion-variables, we needed a set-up where the users could share the environment with a large number of robots, in such a way to replicate a proximal interaction task, as we want to test if the motion-variables really affect this type of interactions. For the complexity of creating such a scenario in a real set-up and also because it is a preliminary inquiry, we chose to create a virtual reality environment. We built the virtual scenario in Unity, which is a multi-platform development software, and we used the Oculus Rift to allow the users to have an immersive experience during the experiment. In our previous work [12] we have demonstrated that virtual reality is a useful tool to replicate interaction experience, especially for preliminary tests.

The scenario was a large open space $(22 \times 11 \mathrm{~m})$ where three groups of robots, each constituted by 20 robots and discernible by the different color, moved near the user (Fig. 1). The robots were simple omnidirectional wheeled robot, with cylindrical shape and a radius equal to $0.1 \mathrm{~m}$. The robots could move freely in the space (namely, no obstacles are considered) and in every trial each group moved towards 
one of the seven goals placed in the environment. The goals were represented by a series of cubes.

During the experiment, the user had to understand the destination of every groups of robots. To provide the answer, she/he used the Oculus Remote (Fig. 1(b)) combined with her/his gaze. When the user felt sufficiently confident about the answer, she/he could select the dedicated object in order to activate the interface where she/he should choose the goals for every group (Fig. 1(c)).

\section{Main experimental factors}

To study the relevance of a series of variables, called independent variables, over certain characteristics, which are the dependent variables, a specific experimental plan must be followed. This plan can be described by a factorial plan [13], where the values of the independent variables are defined for each trial. In particular, we decided to investigate a full factorial plan with three independent variables and each had two possible values, which leads to $2^{3}$ trials. This type of plan has allowed us to investigate the main effect, namely the influence of the motion-variables, and also of the interactions between them.

In order to isolate, as much as possible, the effect of the independent variables over the response, it was important to reduce the disturbing factors that can influence the results. In the following, we report these factors and how we acted in order to lower their effects.

1) Point of view: Since the experiments were carried out in a virtual reality set-up, it was important to consider how the user could move in the environment. Specifically, we chose to forbid any movement of the user apart from the possibility of looking around. This is a good trade-off, given our previous experience with such type of experiments, because it does not cause motion sickness [14] and it does not introduce too much variability.

In addition, in order to recreate a faithful reproduction of a proximal interaction task, we chose to position the user in a neutral location with respect to the robots. As can be retrieved in Fig 1(a), the user was placed among the goals and the robots and at a height that corresponded approximately to a standing position.

2) Position of the goals: To faithfully reproduce the proximal interaction, we positioned the seven goals as reported in Fig. 1(a), in such a way to replicate a scenario where the robots move around the user, but without moving behind her/him. The goals in every trial were randomized for each group.

In [10] the study was conducted considering only two goals, but we think that, in the case of a multi-robot system, and even more in the case of multiple groups, it is more accurate to consider multiple goals. In fact, a multi-robot system is particularly useful when there are multiple tasks to be achieved at the same time. The two external goals were never used because they were not equally probable, namely they could be only confused with one neighbor while the others could be confused with two neighbors.
3) Learning effect: The learning effect represents the fact that, in the first trials, the user can make some mistakes due to the fact that she/he has never used the input method or she/he has not understood some parts of the experiment. On the other side, in the last trials the user could be facilitated by the fact that she/he has already seen the movement in the other trials and hence she/he learned how to read the movement. To reduce this effect the combinations were randomized.

4) Movements of the robots: As the movement of the robots was the core of the experiments, namely the difference between the trials, we saved all the possible combinations offline and then we replicated them in every experiment. This improved the repeatability of the experiments, because every user could see the same motion pattern.

\section{Robot model and independent variables}

The independent variables of the experiments are the motion-variables (trajectory, dispersion and stiffness), each defined on two levels in such a way to make the difference recognizable by the user. The multi-robot system is composed by $N$ mobile robots, each one modeled as a dynamic system in an $n$-dimensional space:

$$
M_{i} \ddot{x}_{i}=w_{i} \quad i=1, \ldots, N
$$

where $M_{i} \in \mathbb{R}^{n \times n}$, positive definite, and $x_{i} \in \mathbb{R}^{n}$ are the inertia matrix and the position of the $i$-th robot, respectively. The control input and all the external forces that act on the robot are included in the term $w_{i} \in \mathbb{R}^{n}$. For simplicity of notation, we will hereafter consider $M_{i}=m I$, where $I \in$ $\mathbb{R}^{n \times n}$ is the identity matrix and $m$ is the mass of the robot.

The motion of the robots is controlled by means of a potential field that implements a cohesive behavior and avoids collisions among the robots. Moreover, it allows to introduce a virtual agent, namely a fictional robot, that follows a given trajectory with a desired velocity's profile, and then to link this virtual agent to the desired group of robots. In this way each group can follow a different trajectory, avoiding collisions and creating a cohesive behavior among the robots belonging to the same group.

1) Trajectory: The trajectory variable represents the position, in every instant of time $t$, of a point that follows a $n$-dimensional path $\varphi(t)$ from a start point $\varphi(t)=\varphi(0)$ to a final point $\varphi(t)=\varphi(f)$, with $f>0$. Hence, inside the trajectory variable, both the geometric path followed by the virtual agent and the velocity's profile with which it is followed are contained.

We investigated the influence of two of the infinite possible trajectories that link two points in an $n$-dimensional space: minimum-jerk and arc-trapezoidal.

The minimum-jerk trajectory is a particular trajectory that minimizes the square of the magnitude of the jerk, which is the rate of change of the acceleration. This type of trajectory can be considered as the ideal candidate to improve legibility because, in [15], it has been shown to be usually used by humans in the act of grasping an object and, in [16], it has been proven to simplify the interaction between a user and a manipulator. The minimum-jerk trajectory, followed by the 
virtual agent, can be described, in the 1-dimensional case, as follows [15]:

$$
\varphi(t)=\varphi(0)+(\varphi(f)-\varphi(0))\left(10\left(\frac{t}{f}\right)^{3}-15\left(\frac{t}{f}\right)^{4}+6\left(\frac{t}{f}\right)^{5}\right)
$$

This can be easily adapted to an $n$-dimensional trajectory, applying (6) componentwise.

On the other hand, the second trajectory has a standard trapezoidal velocity's profile, commonly used in industrial application, and follows the animation principle of "Slow In and Out" and "Arcs" from [17]. The direction, counterclockwise or clockwise, is random.

Both trajectories start and end from and to the same position and the virtual agent takes the same amount of time (60 s) to follow them. The travel time corresponds to the time that the user has available to answer and it is limited because the experiment in a virtual reality set-up could not last a big amount of time (i.e., not more than 10 minutes, from our previous experience).

2) Dispersion and stiffness: The implemented control architecture based on potential fields consists in three different types of potential field: attractive among the members of the same group, attractive between the virtual agent and its respective group and repulsive among every robot, to avoid collisions.

To describe each potential field, it is useful to rewrite (5) considering only the $i$-th robot and explicitly writing the terms that define the control input $\omega_{i}$ :

$$
M_{i} \ddot{x}_{i}=-B_{i} \dot{x}_{i}-\nabla V_{i}
$$

where $\dot{x}_{i} \in \mathbb{R}^{n}$ is the velocity of the $i$-th robot and $B_{i} \in$ $\mathbb{R}^{n \times n}$, positive definite, is the damping factor (which should be introduced for a smooth movement, which has been found to be an important factor when designing an interaction task with mobile robots [8]). We consider a homogeneous friction coefficient along all directions, hence $B_{i}=b I$. The term $\nabla V_{i}$ is the gradient of the potential field $V_{i}$ applied to the $i$-th robot. The potential field $V_{i}$ is described by ${ }^{2}$ :

$$
V_{i}=\sum_{j=1 ; j \neq i}^{N} V_{a_{i, j}}\left(x_{i}, x_{j}\right)+V_{v_{i}}\left(x_{i}, x_{v}\right)+\sum_{j=1 ; j \neq i}^{N} V_{r e p_{i, j}}\left(x_{i}, x_{j}\right)
$$

where $V_{a_{i, j}}$ represents the attractive potential between the $i$-th robot and the $j$-th $\operatorname{robot}^{3}$ :

$$
V_{a_{i, j}}= \begin{cases}\frac{1}{2} K_{a_{i, j}}\left(d_{i j}-d_{0}\right)^{2} & \text { if } d_{i j} \leq d_{1} \\ 0 & \text { otherwise }\end{cases}
$$

Then $V_{v_{i}}$ is the attractive potential between the $i$-th robot and the virtual agent $v$ (it is worth saying that the potential field acts only on the real robots, while the virtual agent follows the given trajectory without feeling any force caused by the

\footnotetext{
${ }^{2}$ In the following formulation we omit the dependence on time of the variables, e.g., $V_{i}(t)=V_{i}$, for ease of notation.

${ }^{3} d_{i j}=\left\|x_{i}-x_{j}\right\|$ is the Euclidean distance between the $i$-th and the $j$-th robot
}

other robots):

$$
V_{v_{i}}= \begin{cases}\frac{1}{2} K_{v_{i, v}}\left(d_{i v}-d_{0_{v}}\right)^{2} & \text { if } d_{i v} \leq d_{1_{v}} \\ 0 & \text { otherwise }\end{cases}
$$

Finally, we have the repulsive potential that acts among all the robots, but not with the virtual agents:

$V_{r e p_{i, j}}=\left\{\begin{array}{cl}K_{r e p_{i, j}}\left(\frac{1}{3} d_{i j}^{3}-d_{m i n}^{3} \ln d_{i j}-\frac{1}{3} d_{m i n}^{3}+\right. & \\ \left.+d_{m i n}^{3} \ln d_{m i n}\right)^{2} & \text { if } d_{i j} \leq d_{m i n} \\ 0 & \text { otherwise }\end{array}\right.$

In all the potentials it is possible to retrieve a positive constant, respectively $K_{a_{i, j}}, K_{r e p_{i, j}}$ and $K_{v_{i, v}}$, that can be used to tune the balance between every action. Parameters $d_{1}, d_{\min }$ and $d_{1_{v}}$ represent the distance at which the $i$-th robot is affected by the corresponding potential field: this recreates the fact that, in a real scenario, the robots have a limited range of communication and a limited range of sensing, hence the robots can not feel objects that are too far away from them.

Dispersion is represented by the desired distance $d_{0}$ in the attractive potential field among the robots belonging to the same group. The Small (-) level of this independent variable is approximately equal to ten times the dimension of one robot $(\approx 0.1 \mathrm{~m}$ of diameter), while the Large $(+)$ level is almost equal to forty times.

Stiffness is defined by the constants $K_{a_{i, j}}$ and $K_{r e p_{i, j}}$ into two levels (Soft (-) and Hard (+)). It sets the strength of the attraction and repulsion force among the robots.

The other parameters used in the computation of the potential fields are equal for all the levels of both variables. Tables I and II report all the values employed during the experiments, which were tuned empirically after preliminary trials.
TABLE I: Dispersion and stiffness levels.

\begin{tabular}{clcc}
\hline & & - & + \\
\hline$d_{0}$ & {$[\mathrm{~m}]$} & 1 & 4 \\
\hline$K_{a_{i, j}}$ & {$[\mathrm{~N} / \mathrm{m}]$} & 0.1 & 1 \\
\hline$K_{r e p_{i, j}}$ & {$[\mathrm{~N} / \mathrm{m}]$} & 1 & 10 \\
\hline
\end{tabular}

TABLE II: Constant variables.

\begin{tabular}{clc}
\hline$K_{v_{i, v}}$ & {$[\mathrm{~N} / \mathrm{m}]$} & 30 \\
\hline$m$ & {$[\mathrm{~kg}]$} & 0.01 \\
\hline$b$ & {$[\mathrm{Ns} / \mathrm{m}]$} & 10 \\
\hline$d_{1}$ & {$[\mathrm{~m}]$} & 5 \\
\hline$d_{1_{v}}$ & {$[\mathrm{~m}]$} & 10 \\
\hline$d_{\text {min }}$ & {$[\mathrm{m}]$} & 0.8 \\
\hline$d_{0_{v}}$ & {$[\mathrm{~m}]$} & 0.1 \\
\hline
\end{tabular}

Fig. 2 represents some examples of the trajectory of the robots. The positions, previously saved offline, have been then used directly in the Unity program to replicate the exact behavior.

\section{E. Dependent variables}

We chose to consider, as dependent variables, the number of correct goals detected in each trial and the time the 


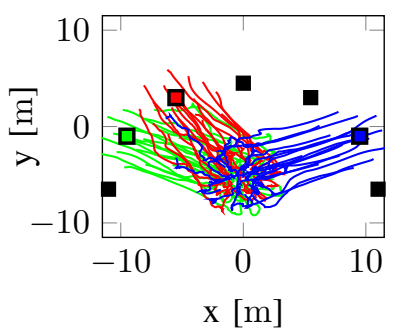

(a) $\mathcal{T}=$ Minimum-jerk, $\mathcal{D}=$ Large, $\mathcal{S}=$ Hard .

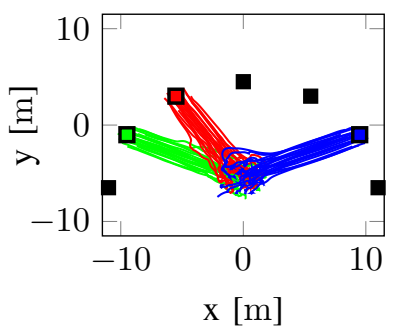

(c) $\mathcal{T}=$ Minimum-jerk, $\mathcal{D}=$ Small, $\mathcal{S}=$ Soft .

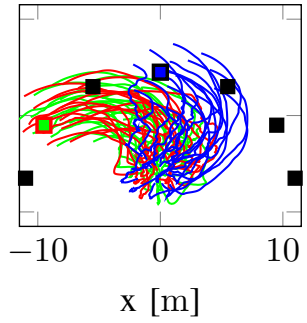

(b) $\mathcal{T}=$ Arc-trapezoidal, $\mathcal{D}=$ Large, $\mathcal{S}=$ Hard .

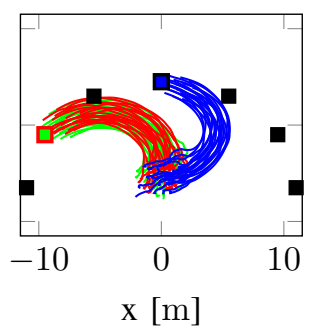

(d) $\mathcal{T}=$ Arc-trapezoidal, $\mathcal{D}=$ Small, $\mathcal{S}=$ Soft .

Fig. 2: Some examples of robots' movement in different trials: each line represents the trajectory of a robot and the color represents the robot's group. The squares represent the goals.

user took to answer. Both variables are important in a collaborative task because the user needs to understand the intentions of the robots, but also to understand them in the fastest possible way. Hence, we have declined the legibility of a multi-robot system into correctness and response time.

\section{F. Statistical analysis}

To analyze the relevance of the motion-variables over the legibility of the system, a 3-way and a 2-way ANOVA were carried out. In both analysis we tested the null hypothesis over the dependent variable:

HO - Null hypothesis: there is no relation between the motion-variables and the legibility of the system (correctness and response time).

In general, a $n$-way ANOVA allows to discover if any effect exists between the $n$ independent variables and the dependent ones, which can be approximated by a random distribution in the case of validation of the null hypothesis. In fact, both variables (correctness and time response), can assume continuous values.

\section{G. Users}

The study was conducted over 15 users (age $26.73 \pm 3.9$, 5 females and 10 males). None was involved in the design of the experiments and they all were new to the trials. They are students and researchers of our engineering department. At the beginning of the experiment they tried the input method inside an example environment, in such a way to further reduce the learning effect, and they signed a consent form. Moreover, they were instructed about the purpose of the experiment and about the working principles of the trials.

Hence, they knew that: 1) in each trial the variables that defined the motion of the robots would be changed, 2) the goals were all equally probable (even thought, as stated in Section III-C.2, the external ones were never selected), and 3) all the robots belonging to a certain group moved towards a goal, never changing their destination.

We chose to replicate, for every user, all the combinations of the motion-variables, applying the within-subjects methodology. All the users stated that they had already tried virtual reality, hence we can state, with a sufficient grade of certainty, that the results are not influenced by the novelty of the means used to create the experiments.

\section{RESUlts}

The dependent variables, correctness and response time, are analyzed with a statistical analysis and, in the following, the results are presented. A few representative trials of the experiments are shown in the attached video.

\section{A. Correctness}

Correctness is represented by the number of correct answers that each user gave. The 3-way ANOVA over the motion-variables outlines that trajectory $(\mathcal{T})$ and dispersion $(\mathcal{D})$ are the main effects that statistically influence the correctness. While the only interaction that results significant is trajectory-dispersion $(\mathcal{T} \mathcal{D})$.

TABLE III: P-value from the 3-way ANOVA.

\begin{tabular}{cc}
\hline Variable & P-value \\
\hline $\mathcal{T}$ & $<0.0001$ \\
\hline $\mathcal{D}$ & 0.0004 \\
\hline $\mathcal{S}$ & 0.4107 \\
\hline $\mathcal{T} \mathcal{D}$ & 0.0039 \\
\hline $\mathcal{T S}$ & 0.2906 \\
\hline $\mathcal{D S}$ & 0.7241 \\
\hline $\mathcal{T} \mathcal{D S}$ & 0.9063 \\
\hline
\end{tabular}

TABLE IV: P-value from the 2-way ANOVA.

\begin{tabular}{cc}
\hline Variable & P-value \\
\hline $\mathcal{D}$ & 0.2924 \\
\hline $\mathcal{S}$ & 0.0001 \\
\hline $\mathcal{D S}$ & 0.0619 \\
\hline
\end{tabular}

Table III reports the p-value of all the main effects and also of the interaction variables. In particular, the p-value represents the smallest level of significance at which the null hypothesis would be rejected. A smaller p-value corresponds to a stronger evidence in favor of the alternative hypothesis. This means that we can reject the null hypothesis for the variables $\mathcal{T}, \mathcal{D}$ and $\mathcal{T} \mathcal{D}$, namely we can assess that these variables influence the correctness. The ANOVA was carried out with a significance level equal to 0.05 , hence the results can be stated with a confidence equal to $95 \%$. In fact all the p-values in Table III smaller than 0.05 allow to reject the null hypothesis.

As this study is only a preliminary inquiry it is difficult to retrieve a function that links the answer with the independent variables. However, for the trajectory variable the mean of 


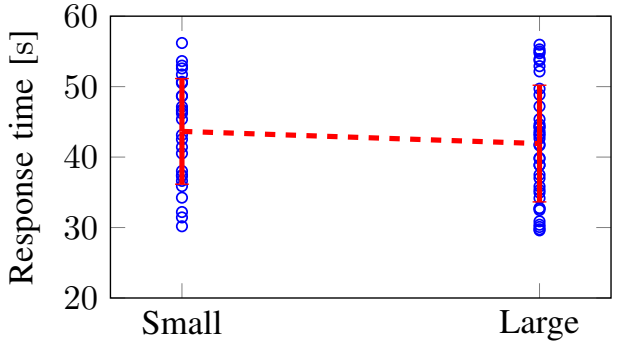

(a) Dispersion level:

Small: $\mu_{T}=43.6, \sigma_{T}=7.5$

Large: $\mu_{T}=41.9, \sigma_{T}=8.3$

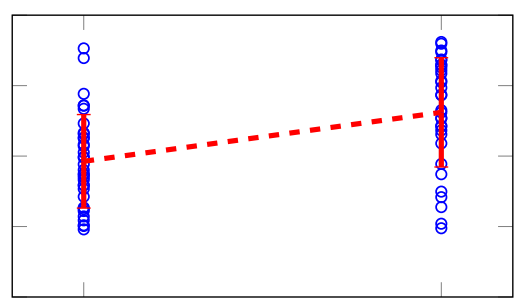

Soft
Hard

(b) Stiffness level:

Soft: $\mu_{T}=39.3, \sigma_{T}=6.6$

Hard: $\mu_{T}=46.2, \sigma_{T}=7.7$

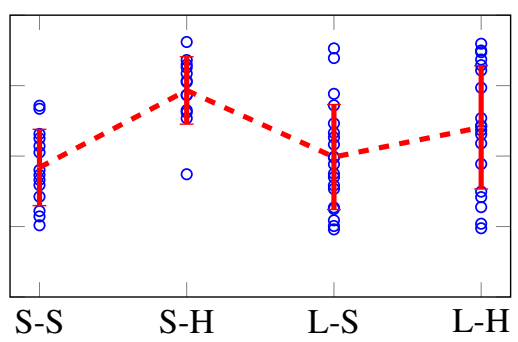

(c) Dispersion-Stiffness level: Small-Soft: $\mu_{T}=38.4, \sigma_{T}=5.4$ Small-Hard: $\mu_{T}=49.3, \sigma_{T}=4.8$ Large-Soft: $\mu_{T}=39.8, \sigma_{T}=7.4$ Large-Hard: $\mu_{T}=44.1, \sigma_{T}=8.7$

Fig. 3: Response time analysis (in blue scatter plot of the trials, in red the mean $\left(\mu_{T}\right)$ and the standard deviation $\left(\sigma_{T}\right)$ of each level).

correct answers for the minimum-jerk is 1.47 and for the arctrapezoidal is 0.35 . The difference is such that we can state that the minimum-jerk trajectory outperforms the other one. This confirms the results obtained in the case of a single group of robots [11].

\section{B. Response time}

The response time is analyzed using only the data of the trials where the user answered correctly for all the groups. This choice is motivated by the fact that the interaction should be effective, rather than quick. To analyze the response time, a penalty function is usually used to consider the wrong answers, or the errors, of the user [18]. But in this case it is not suitable because we should not alter the results before analyzing it in a statistical manner. The analyzed variables are dispersion $(\mathcal{D})$ and stiffness $(\mathcal{S})$ (and their interaction $(\mathcal{D S})$ ). The trajectory variable is excluded because the data were too unbalanced in favor of the minimum-jerk trajectory.

From Table IV we can state that $\mathcal{S}$ is the only motionvariable that affects the response time. In fact, it is the only one with a p-value smaller than 0.05 . However, the interaction variable $\mathcal{D S}$ has a p-value pretty close to the minimum significance level and, as can be retrieved in Fig. 3(c), the mean value of the different combinations of $\mathcal{D S}$ are visually different. Comparing the graphs in Fig. 3 with the same graphs in [11] it is possible to find the same trends. However, the slope of the lines is less pronounced, especially for $\mathcal{D}$ and $\mathcal{D S}$ : this is directly connected to the fact that the two variables are not significant for the response time. If we consider the mean values of the levels of the significant variable $\mathcal{S}$, we can say that the best configuration is the Soft one.

The statistical analysis reports that the considered motionvariables influence significantly the legibility of a multirobot system, also in the case of multiple groups. From the preliminary analysis reported above we can assert that the best combination is minimum-jerk for the trajectory variable, Large for dispersion and Soft for stiffness. This combination is also one of the best for the single group configuration that we have already studied [11].

However, to define a more precise relation between the motion-variables and legibility, a wider factorial plan is needed. In particular, it would be interesting to consider more levels for each variables, except for the trajectory variable for which it is quite clear that it is better choose a path that links directly the starting point to the ending point, instead of one that follows a curve.

\section{CONClusions}

In this paper, we studied the influence of a set of motionvariables upon the legibility of a multi-robot system, specifically divided in multiple groups. The motion-variables are characteristics of the motion of the robots and they are: trajectory, dispersion and stiffness. What we want to discover is if these motion-variables statistically affect the legibility of a multi-robot system constituted by multiple groups. This type of communication allows to interact with robots without any additional device, which we believe shall improve the overall interaction experience.

Trajectory and dispersion were found relevant for the correctness of the answer, while stiffness was found relevant for the response time. These results are retrieved from a statistical analysis carried on a series of data coming from an experimental campaign. The experiments were developed in a virtual reality set-up.

To improve this work we can consider different characteristics of motion, such as formation, initial configuration, direction of movement of the robots and also heterogeneity. Moreover, it would be interesting to remove the color from the robots, making their membership to a certain group indistinguishable, and analyze if it is possible to infer the membership to a group through the motion of the robots.

\section{REFERENCES}

[1] A. Kolling, P. Walker, N. Chakraborty, K. Sycara, and M. Lewis, "Human interaction with robot swarms: A survey," IEEE Transactions on Human-Machine Systems, vol. 46, no. 1, pp. 9-26, 2016. 
[2] A. Franchi, C. Secchi, H. I. Son, H. H. Bulthoff, and P. R. Giordano, "Bilateral teleoperation of groups of mobile robots with time-varying topology," IEEE Transactions on Robotics, vol. 28, no. 5, pp. 10191033, 2012.

[3] L. Sabattini, C. Secchi, B. Capelli, and C. Fantuzzi, "Passivity preserving force scaling for enhanced teleoperation of multirobot systems," IEEE Robotics and Automation Letters, vol. 3, no. 3, pp. 1925-1932, 2018.

[4] C. Secchi, S. Stramigioli, and C. Fantuzzi, Control of interactive robotic interfaces: A port-Hamiltonian approach. Springer Science \& Business Media, 2007, vol. 29.

[5] S. MohaimenianPour and R. Vaughan, "Hands and faces, fast: Monocamera user detection robust enough to directly control a uav in flight," in Proc. 2018 IEEE/RSJ Int. Conf. on Intelligent Robots and Systems (IROS). IEEE, 2018, pp. 5224-5231.

[6] S. Pourmehr, V. M. Monajjemi, R. Vaughan, and G. Mori, “'YYou two! Take off!": Creating, modifying and commanding groups of robots using face engagement and indirect speech in voice commands," in Proc. 2013 IEEE/RSJ Int. Conf. on Intelligent Robots and Systems. IEEE, 2013, pp. 137-142.

[7] P. A. Lasota, T. Fong, J. A. Shah et al., "A survey of methods for

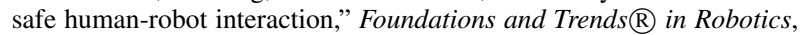
vol. 5, no. 4, pp. 261-349, 2017.

[8] G. Dietz, P. Washington, L. H. Kim, S. Follmer et al., "Human perception of swarm robot motion," in Proc. 2017 CHI Conference Extended Abstracts on Human Factors in Computing Systems. ACM, 2017, pp. 2520-2527.

[9] D. Szafir, B. Mutlu, and T. Fong, "Communication of intent in assistive free flyers," in Proc. 2014 ACM/IEEE Int. Conf. on Human-Robot Interaction (HRI). ACM, 2014, pp. 358-365.

[10] A. D. Dragan, K. C. Lee, and S. S. Srinivasa, "Legibility and predictability of robot motion," in Proc. 8th Annu. ACM/IEEE Int. Conf. on Human-Robot Interaction (HRI). IEEE Press, 2013, pp. 301-308.

[11] B. Capelli, V. Villani, C. Secchi, and L. Sabattini, "Understanding multi-robot systems: on the concept of legibility," in Proc. 2019 IEEE/RSJ Int. Conf. on Intelligent Robots and Systems (IROS). IEEE, 2019.

[12] V. Villani, B. Capelli, and L. Sabattini, "Use of virtual reality for the evaluation of human-robot interaction systems in complex scenarios," in Proc. 27th IEEE Int. Symp. on Robot and Human Interactive Communication (RO-MAN). IEEE, 2018, pp. 422-427.

[13] D. C. Montgomery, Design and analysis of experiments. John wiley \& sons, 2017.

[14] B. Keshavarz, H. Hecht, and B. D. Lawson, "Visually induced motion sickness: causes, characteristics, and countermeasures," in Handbook of virtual environments. CRC Press, 2014, pp. 652-703.

[15] T. Flash and N. Hogan, "The coordination of arm movements: an experimentally confirmed mathematical model," Journal of neuroscience, vol. 5, no. 7, pp. 1688-1703, 1985.

[16] S. Glasauer, M. Huber, P. Basili, A. Knoll, and T. Brandt, "Interacting in time and space: Investigating human-human and human-robot joint action," in Proc. 19th Annu. IEEE Int. Symp. in Robot and Human Interactive Communication (RO-MAN). IEEE, 2010, pp. 252-257.

[17] J. Lasseter, "Principles of traditional animation applied to $3 \mathrm{~d}$ computer animation," in Proc. 1987 ACM Siggraph Computer Graphics, vol. 21, no. 4. ACM, 1987, pp. 35-44.

[18] B. Busch, J. Grizou, M. Lopes, and F. Stulp, "Learning legible motion from human-robot interactions," International Journal of Social Robotics, vol. 9, no. 5, pp. 765-779, 2017. 